\title{
Mutations including the promoter region of myocilin/TIGR gene
}

\author{
Maria Saura ${ }^{1}$, Montse Cabana ${ }^{2}$, Carmen Ayuso ${ }^{3}$ and Diana Valverde ${ }^{* 1}$ \\ ${ }^{1}$ Area de Genética, Facultad de Ciencias, Lagoas-Marcosende 36200, Universidad de Vigo, Spain; ${ }^{2}$ Servicio de \\ Oftalmología, Complejo Hospitalario Juan Canalejo, La Coruña, Spain; ${ }^{3}$ Servicio de Genética. Fundación \\ Jiménez-Díaz, Madrid, Spain
}

Mutations in the MYOC/TIGR gene are responsible for autosomal dominant primary open angle glaucoma (POAG). Almost all mutations responsible for POAG have been detected in the coding region (in particular at exon 3). By using the techniques of PCR, SSCP, automated sequencing and restriction analysis, we have studied 79 patients suffering from glaucoma. We have found five patients with sequence variants in the consensus region of the promoter. These sequence variants might be involved in the altered association between the consensus region and the corresponding transcription factor. This possibility might be favouring the association of other transcription factors, which would operate as activators or inhibitors of the transcription, altering the MYOC/TIGR expression.

European Journal of Human Genetics (2005) 13, 384-387. doi:10.1038/sj.ejhg.5201299

Published online 13 October 2004

Keywords: myocilin; MYOC/TIGR; human trabecular meshwork; POAG; intracellular accumulation; misfolded proteins; upstream stimulatory factor

\section{Introduction}

Glaucoma encompasses a complex of ocular disease entities characterized by a progressive excavation of the optic nerve head and loss of the visual fields, and is frequently associated with increased intraocular pressure (IOP).

The first gene identified as playing a role in glaucoma was MYOC/TIGR, at the GLC1A locus, located at the chromosomal region $1 \mathrm{q} 23-\mathrm{q} 25 .^{1-3}$ MYOC/TIGR was originally discovered as a protein specifically induced in the human trabecular meshwork (HTM) cells by prolonged dexamethasone treatment ${ }^{4}$ It was later reported to be present in the ciliary body ${ }^{5}$ and in the connecting cilium of the photoreceptor cells. ${ }^{6}$

The genomic organization of the MYOC gene is relatively simple. It consists of three exons (604, 126, 782 bp respectively) separated by two introns and a $5-\mathrm{kb}$

*Correspondence: Dr D Valverde, Department of Genetics, Faculty of Science, University of Vigo, Lagoas Marcosende 36200, Vigo, Spain. Tel: + 3498681 3841; Fax: + 3498681 2556; E-mail: dianaval@uvigo.es Received 26 May 2004; revised 25 August 2004; accepted 25 August 2004 promoter region that contains several regulatory elements. ${ }^{7}$ The myocilin gene encodes a secretory protein of 504 amino acids. ${ }^{4,5}$ The deduced amino-acid sequence shows significant homologies with N-terminal region. Homology with myosin is relatively low (25-29\%). However, the carboxy-terminal domain is $40 \%$ homologous to olfactomedin, a major component of the extracellular matrix of the olfactory neuroepithelium. ${ }^{8}$ It is known that mutations in the MYOC/TIGR gene are involved in approximately $4 \%$ of glaucomatous patients. ${ }^{9}$ To date, most mutations linked to glaucoma are missense mutations, and have been mapped to exon 3, which coincides with the conserved olfactomedin domain. ${ }^{10-14}$ It has been suggested that accumulation of myocilin in the extracellular matrix might contribute to the obstruction of the outflow pathway and result in an elevation of intraocular pressure (IOP). ${ }^{4,15,16}$ However, the MYOC/TIGR gene might be involved in the pathogenesis of glaucoma in patients without mutations in the coding region. ${ }^{7}$ In the present study, we have characterized five mutations in the promoter region of this gene. We hypothesize that the expression of MYOC/TIGR in the eye, and its alterations in 
POAG, are regulated at the level of transcription. We have analysed samples from 79 glaucomatous patients, using 109 healthy controls (218 chromosomes).

\section{Materials and methods}

A complete ophthalmic history and examination was performed including the following data: age of onset or age of diagnosis of glaucoma, visual sharpness, biomicroscopy using a slit lamp, intraocular pressure (IOP) before treatment, gonioscopy, glaucoma medication, study of visual fields and study of optic discs, family and personal background. To be eligible for participation in the research, the subjects had to meet the following requirements: Excavation in the optic disc above 0.3-0.4, visual fields with typical glaucoma alterations, IOP above $21 \mathrm{mmHg}$ before treatment and opened angles grade III or IV.

In this work we have analysed the promoter region, exons 1 and 2, of the MYOC/TIGR gene in 79 Galician patients suffering from POAG and in 109 healthy subjects used as controls.

To perform this study we first extracted DNA from venous blood leukocytes. PCR was carried out in a total volume of $25.0 \mu \mathrm{l}$ containing $0.4 \mathrm{ng} / \mu \mathrm{l}$ of genomic DNA, $0.4 \mathrm{pmol} / \mu \mathrm{l}$ of each primer, $0.2 \mathrm{~mm}$ of each dNTP, $1.5 \mathrm{~mm}$ of $\mathrm{MgCl}_{2}$, and $0.04 \mathrm{U} / \mu \mathrm{l}$ of Taq polymerase (Roche Diagnostics, Manheim, Germany) in a thermocycler (GeneAmp9700, PE Applied Biosystems, Foster City, CA, USA). Each fragment was amplified using primers described by Kubota et al in 1998 under the following conditions: an initial denaturation at $94^{\circ} \mathrm{C}$ for $3 \mathrm{~min}$, followed by 35 cycles of denaturation at $94^{\circ} \mathrm{C}$ for $30 \mathrm{~s}$, annealing at $55^{\circ} \mathrm{C}$ for $30 \mathrm{~s}$, and extension at $72^{\circ} \mathrm{C}$ for $30 \mathrm{~s}$. A final extension at $72^{\circ} \mathrm{C}$ for $3 \mathrm{~min}$ completed the reaction. Amplification was confirmed using ethidium bromide stained $2 \%$ agarose gels, and visualized by means of an UV transilluminator.

The PCR products were analysed by electrophoresis in $12 \%$ polyacrylamide gels under denaturant conditions through the SSCP technique. Gels were silver stained and only those PCR products that demonstrated a variation pattern were used for DNA sequencing, as described below.

The PCR products were column-purified using PROMEGA PCR-purification kits, and bi-directional sequencing was performed using an ABI Prism 310 DNA sequencer. In addition, the sequences were analysed using Pairwise BLAST to determine if there were any changes from the normal sequence available in the database (AB006686).

These changes were confirmed by restriction analysis using the Webcutter to choose the enzyme. The sequence variants were confirmed by restriction analysis.

\section{Results}

We have studied a total of 79 patients (42 male and 37 female patients) suffering from primary open-angle glau-
Table 1 Observed sequence variants

\begin{tabular}{llll}
\hline Nucleotide change & $\begin{array}{l}\text { Consensus } \\
\text { sequence } \\
\text { location }\end{array}$ & $\begin{array}{l}\text { Gain(+) or } \\
\text { loss(-) of } \\
\text { restriction site }\end{array}$ & $\begin{array}{l}\text { Digestion } \\
\text { product } \\
(b p)\end{array}$ \\
\hline$-8 \mathrm{C}>\mathrm{T}$ & AP1 AP2 & Cvil1(+) & $189+68$ \\
$-126 \mathrm{~T}>\mathrm{G}$ & Nf-kB & Nlalll(-) & $257+129$ \\
$-83 \mathrm{G}>\mathrm{A}$ & $\mathrm{E}-\mathrm{Box}$ & Ddel(+) & $120+39$ \\
{$[-77 \mathrm{G}>\mathrm{A} ;-78 \mathrm{G}>\mathrm{C}]$} & E-Box & NlallI(-) & $305+81$ \\
\hline
\end{tabular}

coma (POAG) and 109 healthy controls who were selected from the Galician general population. All patients belong to the Ophthalmologic Service of the Juan Canalejo Hospital in A Coruña.

Patients ages ranged from 31 to 85 years; more than $45 \%$ of them were over 70 years and most of them were between 60 and 70 years when they were diagnosed. Among the related systemic diseases found in our patients, arterial hypertension stands out (25\%), followed by diabetes (18\%) and cardiopathy (18\%). Three patients (4\%) with high myopia related to POAG were also found in the study.

\section{Mutation analysis}

We have identified changes in five patients. We found a heterozygous transition $-8 \mathrm{C}>\mathrm{T}$ in a patient with myopia magna, a heterozygous transversion $-126 \mathrm{~T}>\mathrm{G}$ in a patient whose mother was blind, two heterozygous transitions, $-78 \mathrm{~T}>\mathrm{G}$ and $-77 \mathrm{G}>\mathrm{A}$, in a patient with a family history of glaucoma, and finally the $-83 \mathrm{G}>\mathrm{A}$ polymorphism in two patients and in $11.92 \%$ of controls.

For all changes Webcutter was used to find the best enzyme to perform the restriction analysis (Table 1).

\section{Discussion}

Most of the mutations described in the MYOC gene in patients with POAG are located in exon 3. The mutations in this coding region found in our group of patients are described in Vázquez et al; ${ }^{17}$ they comprise $5 \%$ of the 79 patients studied. No mutations were found in exons I and II.

Different hypothesis have been made, which establish correlations between mutations in the MYOC/TIGR gene and the development of open-angle glaucoma. The first hypothesis claims that mutations in the coding-region of the gene produce stop codons, which become truncated proteins. The second theory claims that mutations in the coding-region produce an amino-acid change, the result being a different protein with nonfunctional conformation. This protein shows a solubility different from that shown by the wild type, ${ }^{18,19}$ and it will accumulate as defective conformation aggregates. This accumulation would be interfering in the normal aqueous flow, and it would produce an increase in IOP, which would cause 


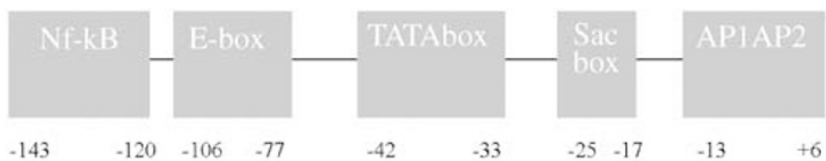

Figure 1 Promoter region of the MYOC gene.

damage to the optic nerve. On the other hand, inmunohistochemical studies have revealed increases in myocilin in the trabecular meshwork in patients without mutations in the coding-region of the MYOC/TIGR gene. Therefore, MYOC gene might be involved in the pathogenesis of open-angle glaucoma in patients showing no mutations in the coding-region. Kirstein et $\mathrm{al}^{7}$ suggest that the gene expression is regulated at transcription level. All sequence variants have been located in the promoter region. The -113 to +67 region of the human MYOC/TIGR promoter contains elements that are required for basal transcription; they also indicate that MYOC/TIGR transcription is very likely to be modulated by additional upstream factors.

We have observed that our sequence variants are located in regulatory regions of the promoter; this fact raises the possibility that the patients suffer from glaucoma due to alterations in the gene expression (Figure 1). One of these changes, $-83 \mathrm{G}>\mathrm{A}^{20}$ has been shown to be associated to the Arg76Lys polymorphism, ${ }^{21}$ but we have not detected it. All the patients showed increased IOP, and needed surgery to control it. Sequence variants located in consensus region might be interfering in the correct union of transcription factors, which can work as activators or inhibitors of transcription. Even if these sequence variants interfered in the correct binding of the transcription factor or favoured the union of another transcription factor, the following conclusions may be drawn.

1. In case they are transcription activators, the action mechanism agrees to Kirstein's purpose. Kirstein et $a l^{7}$ found a group of sequences protected from digestion with DNAse I (cis sequences), which could be involved in expression regulation. These sequences include: TATA box and at least three downstream protein-binding sites, a Sac-box, an AP-1-like sequence and an AP-2-like sequence. Upstream of the TATA box, at least other three protected regions were found. The major footprint was found in a region of 30 protected base pairs from nt -166 to -77 , indicating the presence of either a relatively large binding site or an array of multiple cis elements. The authors concluded that all these factors might play a role in the increase in MYOC expression in eyes with POAG. All the sequence variants found in this work are located in this region.

2. In case they are transcription inhibitors, these mutations would diminish the concentration of the protein myocilin. The myocilin at low concentration might be acting as well as a transcription factor over other proteins. The myocilin gene has a leucine zipper structure characteristic of transcription factors. This would modulate the expression of other proteins, which would cause glaucoma due to incorrect performance.

No changes have been observed in controls apart from the described polymorphism. Expression studies and functional assays may be carried out to determine the role played by these sequence variants as activators or inhibitors of transcription. The results of these studies will shed light on mechanisms for the associations seen and their importance in the development of glaucoma.

\section{References}

1 Sheffield VC, Stone EM, Alward WL et al: Genetic linkage of familial open angle glaucoma to chromosome 1q21-q31. Nat Genet 1993; 4: 47-50.

2 Morissette J, Cote G, Anctil JL et al: A common gene for juvenile and adult-onset primary open-angle glaucomas confined on chromosome 1q. Am J Hum Genet 1995; 56: 1431-1442.

3 Belmouden A, Adam MF, Dupont de Dinechin S et al: Recombinational and physical mapping of the locus for primary open-angle glaucoma (GLC1A) on chromosome 1q23q25. Genomics 1997; 39: 348-358.

4 Nguyen TD, Chen P, Huang WD, Chen H, Johnson D, Polansky JR: Gene structure and properties of TIGR, an olfactomedinrelated glycoprotein cloned from glucocorticoid-induced trabecular meshwork cells. J Biol Chem 1998; 273: 6341-6350.

5 Ortego J, Escribano J, Coca Prados M: Cloning and characterization of subtracted cDNAs from a human ciliary body library encoding TIGR, a protein involved in a juvenile open angle glaucoma with homology to myosin and olfactomedin. FEBS Lett 1997; 413: 349-353.

6 Kubota R, Noda S, Wang Y et al: A novel myosin-like protein (myocilin) expressed in the connecting cilium of the photoreceptor: molecular cloning, tissue expression, and chromosomal mapping. Genomics 1997; 41: 360-369.

7 Kirstein L, Cvekl A, Chauhan BK, Tamm ER: Regulation of human myocilin/TIGR gene transcription in trabecular meshwork cells and astrocytes: role of upstream stimulatory factor. Genes Cells 2000; 5: 661-676.

8 Bal RS, Anholt RR: Formation of the extracellular mucous matrix of olfactory neuroepithelium: identification of partially glycosylated and nonglycosylated precursors of olfactomedin. Biochemistry 1993; 32: 1047-1053.

9 Faucher M, Anctil JL, Rodrigue MA et al: Founder TIGR/myocilin mutations for glaucoma in the Quebec population. Hum Mol Genet 2002; 11: 2077-2090.

10 Sarfarazi M: Recent advances in molecular genetics of glaucomas. Hum Mol Genet 1997; 6: 1667-1677, (review).

11 Rozsa FW, Shimizu S, Lichter PR et al: GLC1A mutations point to regions of potential functional importance on the TIGR/MYOC protein. Mol Vis 1998; 4: 20.

12 Adam MF, Belmouden A, Binisti P et al: Recurrent mutations in a single exon encoding the evolutionarily conserved olfactomedinhomology domain of TIGR in familial open-angle glaucoma. Hum Mol Genet 1997; 6: 2091-2097.

13 Tamm ER, Russell P, Epstein DL, Johnson DH, Piatigorsky J: Modulation of myocilin/TIGR expression in human trabecular meshwork. Invest Ophthalmol Vis Sci 1999; 40: 2577-2582.

14 Fingert JH, Heon E, Liebmann JM et al: Analysis of myocilin mutations in 1703 glaucoma patients from five different populations. Hum Mol Genet 1999; 8: 899-905. 
15 Fingert JH, Ying L, Swiderski RE et al: Characterization and comparison of the human and mouse GLC1A glaucoma genes. Genome Res 1998; 8: 377-384.

16 Fautsch MP, Bahler CK, Jewison DJ, Johnson DH: Recombinant TIGR/MYOC increases outflow resistance in the human anterior segment. Invest Ophthalmol Vis Sci 2000; 41: 4163-4168.

17 Vázquez CM, Herrero OM, Bastus BM, Perez VD: Mutations in the third exon of the MYOC gene in Spanish patients with primary open angle glaucoma. Ophthalmic Genet 2000; 21: 109-115.

18 Tamm ER, Russell P: The role of myocilin/TIGR in glaucoma. Results of the Glaucoma Research Foundation catalyst meeting. Berkeley, CA, March 2000.
19 Caballero M, Borras T: Inefficient processing of an olfactomedindeficient myocilin mutant: potential physiological relevance to glaucoma. Biochem Biophys Res Commun 2001; 282: $662-670$.

20 Fingert JH, Heon E, Liebmann JM et al: Analysis of myocilin mutations in 1703 glaucoma patients from five different populations. Hum Mol Genet 1999; 8: 899-905.

21 Colomb E, Nguyen TD, Bechetoille A et al: Association of a single nucleotide polymorphism in the TIGR/MYOC gene promoter with the severity of primary open angle glaucoma. Clin Genet 2001; 60: 220-225. 